\title{
US population norms for the EQ-5D-5L and comparison of norms from face-to-face and online samples
}

\author{
Ruixuan Jiang $^{1}$ (1) M. F. Bas Janssen ${ }^{2,3} \cdot$ A. Simon Pickard ${ }^{4}$
}

Accepted: 19 September 2020 / Published online: 6 October 2020

(c) The Author(s) 2020

\begin{abstract}
Purpose Normative scores (norms) allow for comparisons between population(s) of interest and the general population, which is useful for burden of disease studies and cost-effectiveness analysis. The primary aim of this study was to estimate US visual analogue scale (EQ VAS) and utility-based norms for the EQ-5D-5L using the face-to-face sample. The secondary aim was to compare norms estimated in the face-to-face and online populations.

Methods This study estimated population norms from two general population surveys: (a) face-to-face and (b) online. In these surveys, respondents provided their health state using the EQ-5D-5L health classifier and the EQ VAS. Descriptive statistics, including mean, standard deviation (SD), 95\% confidence interval, and median for the 5L utility and EQ VAS were estimated for each sample and across relevant respondent characteristics to serve as the basis for US EQ-5D-5L norms Results Face-to-face sample respondents $(n=1134)$ were representative of the US adult general population. In this sample, mean (SD) utility decreased with increasing age until age 45 or greater (age 45-54: $0.816(0.249)$ age 55-64: $0.815(0.243)$ age 65-74: $0.824(0.217)$ age $75+: 0.811(0.218)$ ). With increasing age, more problems were reported on all dimensions except anxiety/depression; a smaller proportion of respondents age 65 and older reported problems with anxiety/depression (23.8\%) as compared to the youngest respondents $(42.1 \%)$. Online $(n=2018)$ mean utility and EQ VAS values were consistently lower than the face-to-face sample.

Conclusions The availability of US EQ-5D-5L norms facilitates interpretation and understanding of general population and patient health.
\end{abstract}

Keywords Population norms $\cdot$ Normative values $\cdot$ EQ-5D-5L $\cdot$ Reference values $\cdot$ Health-related quality of life $\cdot$ Patient reported outcomes $\cdot$ Online $\cdot$ Face-to-face

Results previously presented at 2020 EuroQol Academy in Prague, Czech Republic on March 3, 2020.

Electronic supplementary material The online version of this article (https://doi.org/10.1007/s11136-020-02650-y) contains supplementary material, which is available to authorized users.

A. Simon Pickard

Pickard1@uic.edu

1 Center for Observational and Real-World Evidence, Merck, Kenilworth, NJ, USA

2 EuroQol Group, Rotterdam, The Netherlands

3 Section Medical Psychology and Psychotherapy, Department of Psychiatry, Erasmus MC, Rotterdam, The Netherlands

4 Department of Pharmacy Systems, Outcomes, and Policy, University of Illinois At Chicago College of Pharmacy, $833 \mathrm{~S}$ Wood St, Chicago, IL 60612, USA

\section{Introduction}

The EQ-5D is the most widely used generic multi-attribute utility instrument in the world, and it has numerous applications in health care [1]. It is applied in a variety of research and clinical practice settings, such as clinical trials, costutility analysis (CUA), patient surveillance, and population health measurement [2-8]. The EQ-5D was developed as a brief, generic measure of health which includes a health state classifier that has five dimensions: mobility, self-care, usual activities, pain/discomfort, and anxiety/depression. The first version of the EQ-5D had 3 levels of health problems (EQ5D-3L), and more recently in 2011, a more descriptively rich 5-level version (EQ-5D-5L) was introduced which describes 3125 health states $\left(D^{\mathrm{L}}=5^{5}=3125\right)[9,10]$. An index-based utility score can be generated from self-assessments using the descriptive system, by applying a value set based on 
societal preferences for EQ-5D health states. The value set is based on preference choice tasks elicited from the general population. In addition, the health state classifier is also accompanied by the visual analogue scale (EQ VAS), anchored at 0 for "the worst health you can imagine" and 100 for "the best health you can imagine" [9]. Thus, the EQ$5 \mathrm{D}-5 \mathrm{~L}$ can provide two summary scores of health that can inform decision-making: the patient's self-rating of health on the EQ VAS, and an index-based utility score. The latter is used to facilitate the generation of a common metric of health in the form of quality-adjusted life-years (QALYs) in CUA that can inform resource allocation across health care sectors.

Among the numerous applications of the EQ-5D-5L, a set of utility index and EQ VAS benchmark values for the general population, i.e., population reference data or population norms, are useful for comparing burden of disease and as normative reference values [11]. Patient or sample values can be compared to these benchmark values to determine how individuals or groups measure against the general population in decision models, evaluation of clinical programs, assessment of public health in large-scale applications, etc. [6, 11-23]. Although the use of the EQ-5D-5L continues to expand, the US does not yet have population norms for comparison. In 2019, an EQ-5D-5L value set for the US was developed [24]. It was developed utilizing the standardized international protocol recommended by the EuroQol Group and used preferences from an adult sample representative of the US general population. Apart from the valuation of EQ-5D-5L health states, these respondents also self-reported their own health using the EQ-5D-5L and the EQ VAS, allowing for estimation of US EQ-5D-5L population norms [24].

In the past, most studies reporting population norms have been based on data collected in-person as part of population health studies or using mail surveys $[11,12]$. The standardized international protocol for EQ-5D-5L valuation studies was developed for a face-to-face, interviewer-assisted setting to ensure the respondent task comprehension and preserve higher data quality for the challenging preference choice tasks $[25,26]$. The US EQ-5D-5L study used quotabased sampling based on age, gender, race, and ethnicity to ensure representativeness of the sample for the population and examined non-quota-based respondents characteristics like education to support comparability to the general population. A substudy was also conducted that sought to replicate the face-to-face protocol using online panels, applying the same quota-based sampling criteria [27, 28]. The online and face-to-face samples were dissimilar in terms of health, and the online sample was also less comparable to the general population more than the face-to-face sample in terms of non-quota-based characteristics. Thus, the face-toface valuation study was selected as the primary source of population norms for the present study. However, as online data collection continues to gain relevance, there is value in understanding the differences between the two sources of respondents.

The primary aim of this study was to estimate EQ-5D-5L EQ VAS and index-based norms for the US general population using the data from the US valuation face-to-face study. A secondary aim was to compare face-to-face and online samples based on non-quota-based characteristics to understand the issues of generalizability with respect to mode of data collection.

\section{Methods}

\section{Data sources}

\section{EQ-5D-5L face-to-face valuation study}

The purpose of the EQ-5D-5L face-to-face valuation study was to elicit preferences for EQ-5D-5L health states from a representative sample of the US adult general population. This study was granted Institutional Review Board (IRB) exemption by the University of Illinois at Chicago IRB. Collected preferences were used as the basis for modeling the US EQ-5D-5L value set [24]. The face-to-face study followed the internationally standardized EQ-5D-5L valuation study protocol based on a robust body of evidence [25, 29-32]. Respondents were recruited using a variety of inperson, community, and online advertising methods. They were quota-sampled using age, gender, race, and ethnicity to match the most recently available US population parameters at the time. Respondents indicated informed consent to the interviewer prior to survey participation.

Eleven interviewers traveled around the United States between May and August 2017 to survey respondents in oneon-one sessions in six metropolitan areas: Chicago, Philadelphia, Phoenix, Birmingham, Seattle, and Denver [24]. Interview areas were chosen based on their representativeness of the general US population and to ensure data collection in each of the four US census regions: Northeast, Midwest, West, and South. Interview sessions occurred at several locations around each metropolitan area, including both city and suburban/rural areas. Each interview was conducted as a face-to-face, computer-assisted personal interview (CAPI). Surveys were completed in Spanish or English based on the respondent's preferred language.

At the start of these surveys, respondents described their own health using the EQ-5D-5L visual analogue scale (EQ VAS) and the EQ-5D-5L descriptive system by indicating their level of problems on each of the 5 dimensions on the day of the survey (no, slight, moderate, severe, or extreme problems/unable to) [24]. Index-based utility scores for each 
respondent's self-reported EQ-5D-5L health state was estimated by applying the US value set [24]. The US value set was based on the composite time trade-off (cTTO) preferences, and index values ranged from -0.573 for the worst $(55,555)$ to 1 for the best $(11,111)$ EQ-5D-5L health state. Respondent self-reported EQ VAS values were also used to estimate general population norms.

\section{EQ-5D-5L online valuation study}

The EuroQol valuation study protocol and survey were also replicated in an online valuation substudy. The study was determined to be eligible for an IRB exemption by the Western Institutional Review Board. Respondents in the online study were recruited from established online survey panels using the same strata as the face-to-face study. Respondents indicated informed consent on the first survey screen prior to participation. The sequence and content of self-reported respondent characteristics, including EQ VAS and as an EQ-5D-5L health state, were the same between online and face-to-face surveys. Online respondents self-completed the survey without any interviewer supervision.

\section{Analyses}

Norms for the utility index and EQ VAS were estimated using descriptive statistics, including mean, standard deviation, 95\% confidence interval, and median. Proportions of respondents endorsing each level of severity for the five dimensions were also calculated. Norms were computed for the face-to-face and online samples separately. Characteristics important for informing population health, CUA, and clinical outcomes research were included as stratifying variables in the present analyses, including sociodemographic information (age, gender, race, ethnicity), and health (general health status, number of regular prescription medications) [33, 34]. Age was divided into seven age bands (18-24, 25-34, 35-44, 45-54, 55-64, 65-74, 75+).

During the surveys, respondents also self-reported diagnoses of certain illnesses, and the mean utility and EQ VAS values were estimated for each diagnosis. Only illnesses that were self-reported by more than 100 respondents were included in these analyses due to instability of the estimates with small sample sizes.

Utility and EQ VAS were analyzed as continuous variables. Statistical testing for differences in mean VAS or utility across groups was conducted using t-tests (gender, ethnicity, experience with illness) or ANOVA (age, race, general health status, number of prescription medications). Significance was designated at $p<0.05$. All statistical analyses were completed in SAS 9.4 (Cary, NC, USA) The online and face-to-face norms were qualitatively compared.

\section{Results}

\section{US EQ-5D-5L norms}

One thousand one hundred and thirty-four adult respondents were recruited as part of the US face-to-face valuation study [24]. The sample was representative of the US general population for age, gender, race, and ethnicity (Table 1). Compared to the general US population, the face-to-face sample was more likely not to have child dependents under 18 and attained education greater than secondary school. There were no missing data for the EQ5D-5L health states nor the VAS. Some covariates were missing for a single respondent who had to terminate the interview early. Five respondents identified as non-binary for gender, but norms could not be generated due to the limited sample size.

Of the face-to-face sample, $31.2 \%$ of the respondents reported no problems on any of the EQ-5D-5L dimensions (11,111). (Table 2) The mean (standard deviation) utility value for the face-to-face sample was 0.851 (0.205). (Table 3 ) Mean utility differed across age groups $(p<0.001)$ and decreased with increasing age until the 45-54 age band. Means for age bands 45-54 and 55-64 were similar $-0.816(0.249)$ and $0.815(0.243)$, respectively. The mean increased for age band 65-74: 0.824 (0.217) and decreased again for the oldest age band of 75+: $0.811(0.218)$. Women and men had similar mean utility scores: $0.856(0.191)$ versus $0.847(0.219)$ $(p=0.487)$. No statistically significant differences in mean utility scores were identified across race and ethnicity categories.

Mean utility scores decreased with poorer general health as respondents with excellent, very good, good, fair, and poor health had mean (SD) scores of $0.951(0.096)$, 0.910 (0.131), 0.835 (0.160), 0.632 (0.262), and 0.338 (0.380), respectively. (Table 3 ) The mean utility also decreased with increasing number of regular prescriptions taken $(p<0.001)$. Respondents with experience with serious illness, whether personal, intra-familial, or caring for others, had lower mean utility and EQ VAS than those with without the experience ( $p<0.01$ for all comparisons).

The mean (SD) EQ VAS for the sample was 80.4 (15.6). (Table 3) The mean EQ VAS pattern across age bands differed from mean index. EQ VAS decreased with increasing age until the 45-54 age band and reached a nadir of 75.9 (18.6); it then increased through the rest of the age bands, reaching 81.1 (15.6) for respondents 75 and older. Across other respondent characteristics, VAS norm trends were comparable to those observed for utility norms.

Of the EQ-5D-5L dimensions, fewest respondents reported problems with self-care $(6.5 \%$ of respondents) 
Table 1 Face-to-face and Online respondent characteristics as compared to the US general population

\begin{tabular}{|c|c|c|c|}
\hline Characteristic & $\begin{array}{l}\text { US general population } \\
(n=327,167,439)\end{array}$ & $\begin{array}{l}\text { Face-to-face sample } \\
(n=1134)\end{array}$ & $\begin{array}{l}\text { Online } \\
\text { sample } \\
(n=2018)\end{array}$ \\
\hline Age, mean (SD), $n(\%)$ & & $46.9(18.1)$ & $45.6(15.5)$ \\
\hline $18-24$ & $12.1 \%$ & $107(9.4)$ & $133(6.6)$ \\
\hline $25-34$ & $17.9 \%$ & $251(22.1)$ & $494(24.5)$ \\
\hline $35-44$ & $16.3 \%$ & $182(16.0)$ & $385(19.1)$ \\
\hline $45-54$ & $16.4 \%$ & $212(18.7)$ & $330(16.4)$ \\
\hline $55-64$ & $16.7 \%$ & $159(14.0)$ & $386(19.1)$ \\
\hline $65-74$ & $12.0 \%$ & $127(11.2)$ & $252(12.5)$ \\
\hline $75+$ & $6.1 \%$ & $96(8.5)$ & $38(1.9)$ \\
\hline \multicolumn{4}{|l|}{ Gender, $n(\%)$} \\
\hline Male & $48.3 \%$ & $564(49.7)$ & $973(48.2)$ \\
\hline Female & $51.4 \%$ & $565(49.8)$ & $1041(51.6)$ \\
\hline Gender, other & $0.3 \%$ & $5(0.4)$ & $4(0.20)$ \\
\hline \multicolumn{4}{|l|}{ Race, $n(\%)$} \\
\hline White & $65.5 \%$ & $685(60.4)$ & $1570(77.8)$ \\
\hline Black & $11.9 \%$ & $152(13.4)$ & $258(12.8)$ \\
\hline Hispanic ethnicity, $n(\%)$ & $15.0 \%$ & $208(18.3)$ & $308(15.3)$ \\
\hline $\begin{array}{l}\text { Education level greater than second- } \\
\text { ary, } n(\%)\end{array}$ & $58.9 \%$ & $732(64.6)$ & $1316(65.2)$ \\
\hline \multicolumn{4}{|c|}{ Child dependents (may choose more than 1) } \\
\hline None & $71.2 \%$ & $916(80.8)$ & $1377(68.2)$ \\
\hline Child(ren), $\leq 5$ years old & - & $68(6.0)$ & $221(11.0)$ \\
\hline Child(ren), 6 to 17 years old & - & $180(15.9)$ & $536(25.6)$ \\
\hline \multicolumn{4}{|l|}{ Primary health insurance } \\
\hline None & $10.3 \%$ & $98(8.6)$ & $211(10.5)$ \\
\hline Public & $35.4 \%$ & $480(42.3)$ & $734(36.4)$ \\
\hline Private & $68.7 \%$ & $555(49.1)$ & $1073(53.2)$ \\
\hline Country of birth, United States & & $983(86.7)$ & $1903(94.3)$ \\
\hline \multicolumn{4}{|l|}{ History of illness, $n(\%)$} \\
\hline Hypertension & $32.0 \%$ & $270(23.8)$ & $507(25.1)$ \\
\hline Arthritis & $22.7 \%$ & $267(23.5)$ & $445(22.1)$ \\
\hline Diabetes & $9.4 \%$ & $111(9.8)$ & $223(11.1)$ \\
\hline Heart failure & $2.2 \%$ & $20(1.8)$ & $28(1.4)$ \\
\hline Stroke & $1.8-2.4 \%$ & $23(2.0)$ & $39(1.9)$ \\
\hline Bronchitis & $3.6 \%$ & $29(2.6)$ & $50(2.5)$ \\
\hline Asthma & $7.5 \%$ & $132(11.6)$ & $195(9.7)$ \\
\hline Depression & $25.7 \%$ & $295(26.0)$ & $438(21.7)$ \\
\hline Migraine & $16.0 \%$ & $164(14.5)$ & $232(11.5)$ \\
\hline Cancer & $5.9 \%$ & $65(5.7)$ & $42(2.1)$ \\
\hline None & - & $372(32.8)$ & $692(34.3)$ \\
\hline \multicolumn{4}{|l|}{ Health status, $n(\%)$} \\
\hline Excellent & $20.0 \%$ & $227(20.0)$ & $245(12.1)$ \\
\hline Very good & $33.6 \%$ & $421(37.2)$ & $695(34.4)$ \\
\hline Good & $31.2 \%$ & $332(29.3)$ & $730(36.2)$ \\
\hline Fair & $12.5 \%$ & $124(10.9)$ & $290(14.4)$ \\
\hline Poor & $2.7 \%$ & $29(2.6)$ & $58(2.9)$ \\
\hline
\end{tabular}

and the most respondents reported any problems with pain/discomfort (51\% of respondents). (Table 4) For both the index score and EQ VAS-based norms across the entire sample, means generally decreased with increasing problems on each dimension. Few respondents endorsed severe (level 4) and extreme problems/unable to (level 
Table 2 Most frequent self-reported EQ-5D-5L health states in the face-to-face sample (frequencies greater than or equal to $0.5 \%$ )

\begin{tabular}{lrllll}
\hline $\begin{array}{l}\text { EQ-5D-5L } \\
\text { health state }\end{array}$ & $N$ & $\%$ & $\begin{array}{l}\text { EQ-5D-5L } \\
\text { health state }\end{array}$ & $N$ & $\%$ \\
\hline 11111 & 354 & 31.2 & 21122 & 10 & 0.9 \\
11121 & 138 & 12.2 & 11133 & 8 & 0.7 \\
11112 & 95 & 8.4 & 11213 & 7 & 0.6 \\
11122 & 64 & 5.6 & 11222 & 7 & 0.6 \\
21121 & 37 & 3.3 & 21211 & 7 & 0.6 \\
21111 & 24 & 2.1 & 21233 & 7 & 0.6 \\
11123 & 19 & 1.7 & 11132 & 6 & 0.5 \\
11113 & 18 & 1.6 & 11223 & 6 & 0.5 \\
21222 & 17 & 1.5 & 11232 & 6 & 0.5 \\
11221 & 14 & 1.2 & 21132 & 6 & 0.5 \\
11131 & 12 & 1.1 & 21223 & 6 & 0.5 \\
21221 & 12 & 1.1 & 31121 & 6 & 0.5 \\
21231 & 12 & 1.1 & 31131 & 6 & 0.5 \\
11211 & 11 & 1 & & & \\
\hline
\end{tabular}

5) on most dimensions. The prevalence of any problems on each EQ-5D-5L dimension increased for all dimensions with advancing age except for anxiety/depression, which had the opposite trend. (Supplementary material Appendix A).

Norms were also estimated separately by gender with additional stratification by age and general health status. (Table 5) Across age bands, women had higher mean index than men except for the 45-54 and 65-74 age bands where the pattern was reversed (45-54: mean (SD) men: 0.825 (0.256) women: 0.807 (0.241); 65-74: men: 0.827 (0.216) women: $0.821(0.221)$ ). The mean index and EQ VAS in both genders consistently decreased with decreasing general health. Men with self-reported excellent health had mean (SD) utility index of $0.942(0.121)$, and those with poor health had $0.258(0.344)$. Mean EQ VAS in men ranged from 91.2 (10.2) to 36.9 (19.7) by general health status. Women with self-reported excellent health had mean utility of $0.962(0.057)$ whereas those with poor health had mean utility of $0.396(0.412)$. Mean EQ VAS in women ranged from 92.2 (8.2) to 56.5 (18.6). Notably, women with poor health reported higher utility and EQ VAS than men with the same general health status.

The self-reported conditions with the greatest effect on health-related quality of life (HRQoL) included depression, arthritis, and sinusitis with mean utility values of 0.708 (0.258), $0.712(0.266)$, and 0.730 (0.279), respectively. (Table 3) The health conditions with the least impact on HRQoL were hypertension: 0.794 (0.223); diabetes mellitus: 0.788 (0.233); and hay fever: 0.785 (0.249).

\section{Online descriptive statistics and comparison to face-to-face norms}

Two thousand and eighteen respondents were recruited from online panels to participate in the online EQ-5D-5L valuation study. Online respondents were also generally representative of the US general population, but the Caucasian race was overrepresented; $77.8 \%$ of online respondents were White whereas only $65.5 \%$ of the US adult general population was White (Table 1). Similar to the face-to-face sample, online respondents were also more likely to have attained at least secondary education compared to the US general population. Respondents who began but did not complete the survey were not included in these analyses. Two thousand and eighteen respondents completed the online survey, and there was no missing data in this sample.

Of the online sample, $23.9 \%$ of the respondents reported no problems on any of the EQ-5D-5L dimensions. (Supplementary material Appendix B) In comparison to faceto-face respondents, online respondents had similar patterns of mean utility and EQ VAS values across different levels of covariates, e.g., decreasing mean norms with worsening general health status and lack of consistent decrease of mean norms with increasing age. (Tables 3-5, Supplementary material Appendices C-E) Online respondents were also more likely to report issues on EQ-5D-5L dimensions with increasing age except for anxiety/depression. (Supplementary material Appendices A, F) Online respondents consistently had lower mean index and EQ VAS values compared to the matching face-to-face age subgroup (Fig. 1). The mean index and EQ VAS values of the overall online sample were $0.800(0.236)$ and $74.6(18.7)$, respectively, whereas the corresponding values in the face-to-face sample were 0.851 (0.205) and 80.4 (15.6). (Table 3 and Supplementary material Appendix C). For both the EQ VAS and the utility index, the corresponding online values were consistently lower than the face-to-face values across age bands and levels of general health. (Fig. 1).

The effect of mode of administration on index and VAS norms were isolated in linear regressions. After controlling for age, race, gender, ethnicity, and general health status, online respondents had average index and VAS values that were 0.027 and 3.0 units lower than face-to-face respondents, respectively. (Results not shown).

\section{Comparison of older adult respondents to the US general population}

As the plateau effect of norms with increasing age was unexpected, additional comparisons of respondents who were at least 65 years old to the US population were completed. Two hundred and twenty-three face-to-face respondents and 290 online respondents were in this age segment (Supplementary 
Table 3 Face-to-face index and VAS-based norms by respondent characteristics

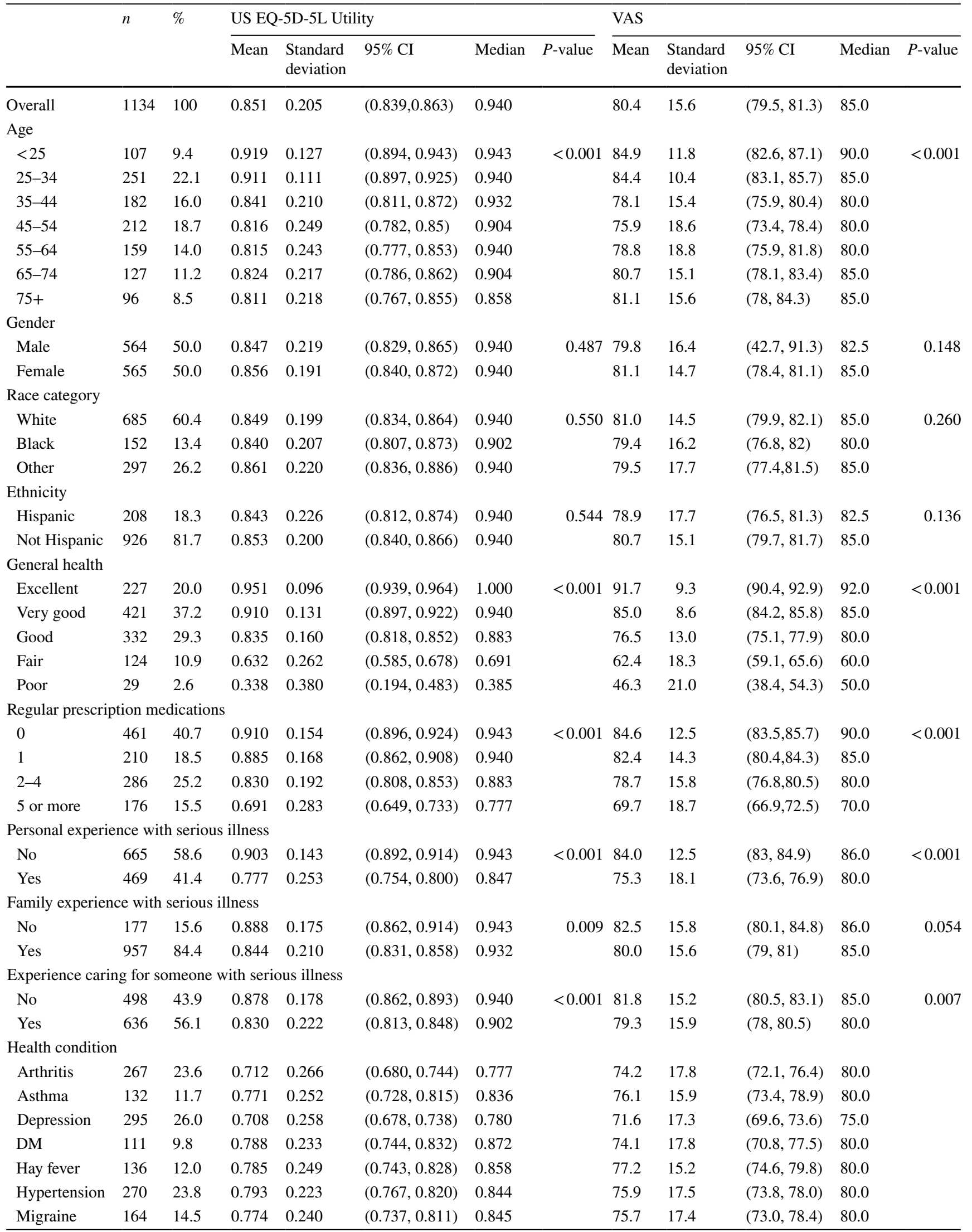


Table 3 (continued)

\begin{tabular}{|c|c|c|c|c|c|c|c|c|c|c|c|}
\hline & \multirow[t]{2}{*}{$n$} & \multirow[t]{2}{*}{$\%$} & \multicolumn{5}{|c|}{ US EQ-5D-5L Utility } & \multicolumn{4}{|l|}{ VAS } \\
\hline & & & Mean & $\begin{array}{l}\text { Standard } \\
\text { deviation }\end{array}$ & $95 \% \mathrm{CI}$ & Median & $P$-value & Mean & $\begin{array}{l}\text { Standard } \\
\text { deviation }\end{array}$ & $95 \% \mathrm{CI}$ & Median $P$-value \\
\hline Sinusitis & 114 & 10.1 & 0.730 & 0.279 & $(0.679,0.782)$ & 0.817 & & 74.2 & 17.3 & $(71.0,77.4)$ & 80.0 \\
\hline
\end{tabular}

material Appendix G). Face-to-face respondents were more similar to the US general population in terms of distribution of age bands, race, and gender. Both sets of respondents were more educated than the US general population-larger portions of face-to-face and online respondents achieved at least a bachelor's degree as compared to the US general population. The face-to-face sample was healthier than the respondents recruited for the Medical Expenditure Panel Survey (MEPS), but the comparison of respondent health between the online sample and MEPS respondents was less straightforward.

\section{Discussion}

In this study, we reported age and gender-based norms for the EQ-5D-5L in the US general population. The reference norms were based on data collected from implementation of the EuroQol international standardized protocol using a study design that used quota-based sampling and involved face-to-face, interviewer-assisted administration of the survey and valuation tasks. Data based on the same quotas for sampling collected via online panels provided systematically different respondents and norms, so these were reported separately.

Index-based utility and EQ VAS mean scores did not consistently decrease with increasing age. Instead, the norms were characterized by plateaus or minor increases in older age bands. This pattern may be explained by the lower reported prevalence of anxiety/depression with increasing age as well as different psychometric properties of the EQ-5D-5L (e.g., differential item functioning) and varying priorities for the dimensions by age [35-41]. In the face-to-face sample, approximately $24 \%$ of respondents 65 and older reported any problems with anxiety/depression whereas $42.1 \%$ of respondents 18 to 24 years old reported the same.(Supplementary material Appendix A) Although issues with mental health may be more often associated with social stigma in older adults and cause fewer older adults to indicate issues with mental health [42], differing levels of social desirability bias due to interviewer presence by age band was unlikely the only contributor to this unexpected distribution by age; the same differential between age bands was observed in the online sample: $24-30 \%$ of online respondents 65 and older and $57.1 \%$ of respondents
18-24 reported any problems with anxiety/depression (Supplementary material Appendix F).

Similar to the norms reported here, other research corroborates higher prevalence of mental health (e.g., anxiety and depression) issues in US young adults [35-38]. In 2017, the National Institute of Mental Health (NIMH) and the Substance Abuse and Mental Health Services Administration (SAMHSA) found that $4.7 \%$ of US adults 50 years and older had a major depressive episode in the past year compared to $13.1 \%$ in respondents 18 to 25 years old [35]. Older data from 2001 to 2003 showed that $9.0 \%$ of respondents 60 years and older had anxiety disorder in the past year whereas $22.3 \%$ of respondents 18 to 29 years old reported the same [36]. Further, mental health reasons for leaving jobs were highest in youngest members of the workforce, and anxiety has also been increasing among young adults since 2008 [37, 38]. In developed, Western countries such as Germany and the UK, some younger segments of the population were also more likely to report mental health issues compared to older segments [43, 44].

Response shift may also have occurred in the older respondents, potentially leading them to interpret or conceptualize the meaning of "no problems" differently from younger respondents who may impose a more ideal expectation when self-reporting their health [39-41, 45, 46]. These interpretation differences could contribute to distinctive psychometric properties of the EQ-5D-5L in different age segments of the population. A combination of increased mental health problems in younger adults and differing interpretation of the problem severity labels could have contributed to the decreased prevalence of anxiety/depression in older respondents and the observed patterns of norms across age groups.

The patterns seen in the US EQ-5D-5L norms are also present in other international norms. Similar to this study, a greater proportion of younger respondents in Chinese urban and Indonesia general populations reported problems with anxiety/depression on the EQ-5D-5L [17, 19]. In China, $34.5 \%$ of men 20 to 29 reported any problems with anxiety/ depression whereas only $11.5 \%$ of men over 70 did the same; the trend was similar in Chinese women [19]. Approximately $40.1 \%$ of Indonesian respondents aged 17 to 30 indicated they had any issues with anxiety/depression compared to $32 \%$ of respondents older than 50 [17]. Similar to the present study, Canadian, specifically Alberta and Quebec, and 


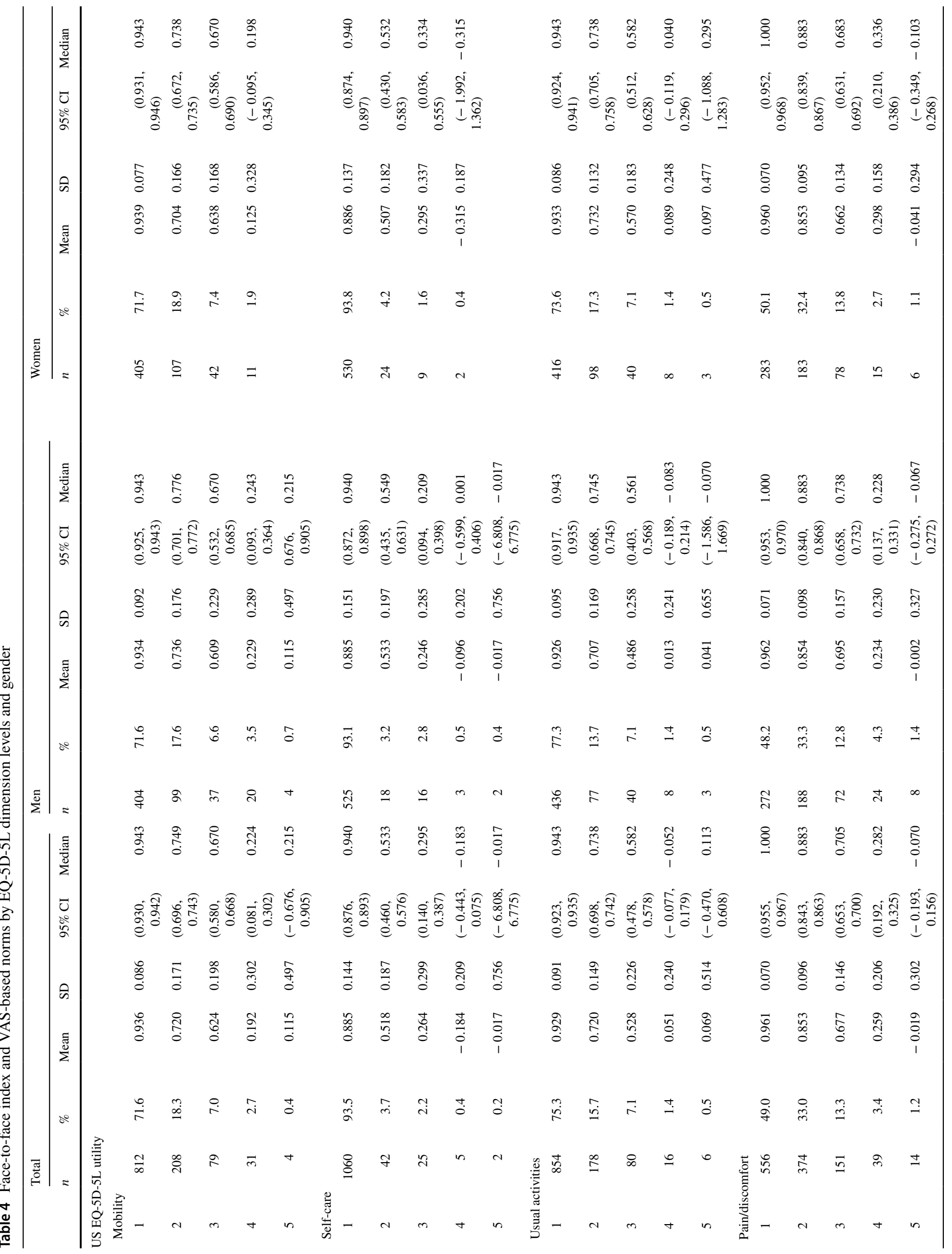




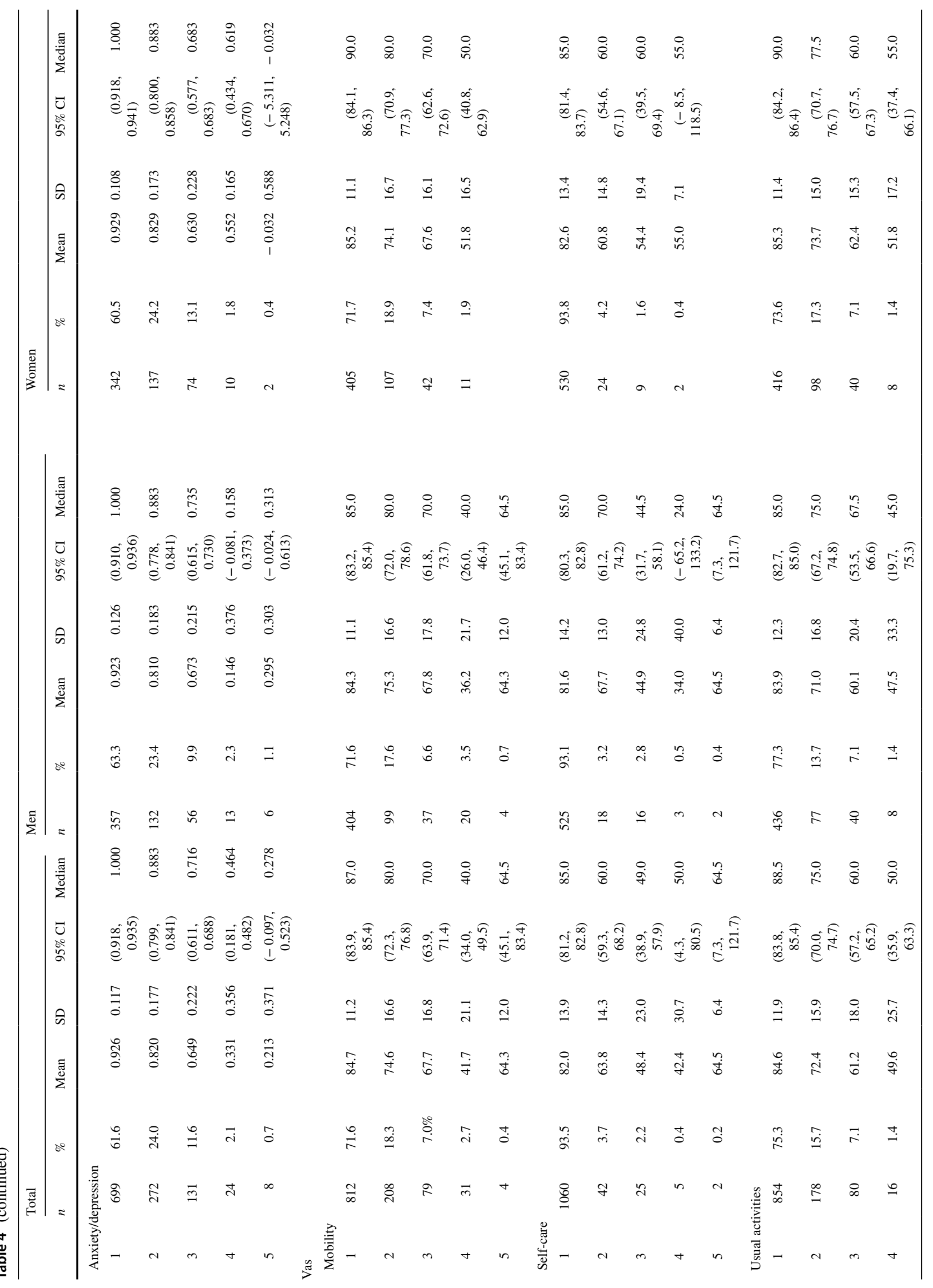


812

Quality of Life Research (2021) 30:803-816

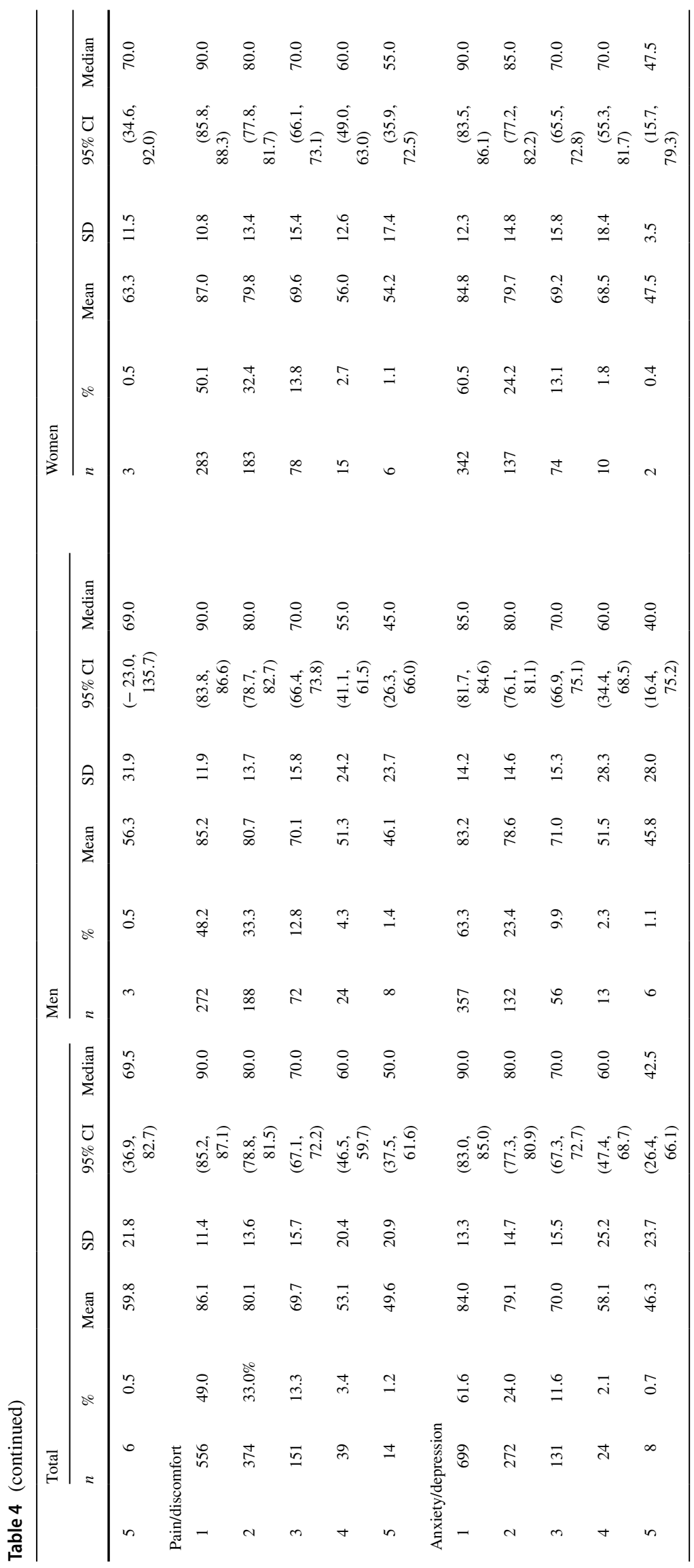

Springer 
Table 5 Face-to-face index and VAS-based norms by age and general health status stratified by gender

\begin{tabular}{|c|c|c|c|c|c|c|c|c|c|c|c|c|}
\hline & \multicolumn{6}{|l|}{ Men } & \multicolumn{6}{|c|}{ Women } \\
\hline & $n$ & $\%$ & Mean & $\begin{array}{l}\text { Standard } \\
\text { deviation }\end{array}$ & $95 \% \mathrm{CI}$ & Median & $n$ & $\%$ & Mean & $\begin{array}{l}\text { Standard } \\
\text { deviation }\end{array}$ & $95 \% \mathrm{CI}$ & Median \\
\hline \multicolumn{13}{|c|}{ EQ-5D-5L index } \\
\hline Overall & 564 & 50.0 & 0.847 & 0.219 & $(0.829,0.865)$ & 0.940 & 565 & 50.0 & 0.856 & 0.191 & $(0.840,0.872)$ & 0.940 \\
\hline \multicolumn{13}{|l|}{ Age } \\
\hline$<25$ & 54 & 9.6 & 0.906 & 0.157 & $(0.863,0.949)$ & 0.943 & 53 & 9.4 & 0.931 & 0.085 & $(0.908,0.955)$ & 0.943 \\
\hline $25-34$ & 118 & 20.9 & 0.907 & 0.114 & $(0.887,0.928)$ & 0.940 & 130 & 23.0 & 0.916 & 0.107 & $(0.897,0.934)$ & 0.943 \\
\hline $35-44$ & 86 & 15.2 & 0.841 & 0.212 & $(0.795,0.886)$ & 0.903 & 95 & 16.8 & 0.845 & 0.209 & $(0.803,0.888)$ & 0.940 \\
\hline $45-54$ & 110 & 19.5 & 0.825 & 0.256 & $(0.777,0.873)$ & 0.940 & 102 & 18.1 & 0.807 & 0.241 & $(0.759,0.854)$ & 0.883 \\
\hline $55-64$ & 91 & 16.1 & 0.807 & 0.266 & $(0.752,0.863)$ & 0.904 & 67 & 11.9 & 0.827 & 0.211 & $(0.776,0.879)$ & 0.940 \\
\hline $65-74$ & 70 & 12.4 & 0.827 & 0.216 & $(0.775,0.878)$ & 0.889 & 57 & 10.1 & 0.821 & 0.221 & $(0.762,0.879)$ & 0.904 \\
\hline $75+$ & 35 & 6.2 & 0.786 & 0.272 & $(0.693,0.88)$ & 0.818 & 61 & 10.8 & 0.825 & 0.181 & $(0.779,0.872)$ & 0.878 \\
\hline \multicolumn{13}{|l|}{ General health } \\
\hline Excellent & 118 & 20.9 & 0.942 & 0.121 & $(0.920,0.964)$ & 1.000 & 109 & 19.3 & 0.961 & 0.057 & $(0.950,0.972)$ & 1.000 \\
\hline Very good & 208 & 36.9 & 0.902 & 0.155 & $(0.881,0.923)$ & 0.940 & 212 & 37.6 & 0.917 & 0.103 & $(0.903,0.931)$ & 0.940 \\
\hline Good & 161 & 28.5 & 0.842 & 0.164 & $(0.816,0.867)$ & 0.883 & 169 & 30.0 & 0.830 & 0.158 & $(0.806,0.854)$ & 0.878 \\
\hline Fair & 62 & 11.0 & 0.641 & 0.279 & $(0.570,0.712)$ & 0.718 & 61 & 10.8 & 0.623 & 0.247 & $(0.560,0.687)$ & 0.688 \\
\hline Poor & 15 & 2.7 & 0.258 & 0.344 & $(0.068,0.449)$ & 0.219 & 13 & 2.3 & 0.396 & 0.412 & $(0.147,0.645)$ & 0.526 \\
\hline \multicolumn{13}{|l|}{ EQ VAS } \\
\hline Overall & 564 & 50.0 & 79.8 & 16.4 & $(78.4,81.1)$ & 82.5 & 565 & 50.0 & 81.1 & 14.7 & $(79.9,82.3)$ & 85 \\
\hline \multicolumn{13}{|l|}{ Age } \\
\hline$<25$ & 54 & 9.6 & 84.4 & 11.7 & $(81.2,87.5)$ & 85.0 & 53 & 9.4 & 85.4 & 11.9 & $(82.2,88.7)$ & 90 \\
\hline $25-34$ & 118 & 20.9 & 84.6 & 10.0 & $(82.8,86.4)$ & 85.0 & 130 & 23.0 & 84.4 & 10.6 & $(82.6,86.2)$ & 85 \\
\hline $35-44$ & 86 & 15.2 & 78.1 & 15.4 & $(74.8,81.4)$ & 80.0 & 95 & 16.8 & 78.6 & 15.0 & $(75.5,81.6)$ & 80 \\
\hline $45-54$ & 110 & 19.5 & 76.2 & 19.4 & $(72.6,79.9)$ & 80.0 & 102 & 18.1 & 75.6 & 17.7 & $(72.1,79.1)$ & 80 \\
\hline $55-64$ & 91 & 16.1 & 76.5 & 20.8 & $(72.2,80.8)$ & 80.0 & 67 & 11.9 & 82.0 & 15.5 & $(78.2,85.8)$ & 85 \\
\hline $65-74$ & 70 & 12.4 & 81.7 & 14.5 & $(78.3,85.2)$ & 85.0 & 57 & 10.1 & 79.6 & 15.8 & $(75.4,83.8)$ & 80 \\
\hline $75+$ & 35 & 6.2 & 76.2 & 18.1 & $(70,82.4)$ & 80.0 & 61 & 10.8 & 84.0 & 13.3 & $(80.6,87.4)$ & 90 \\
\hline \multicolumn{13}{|c|}{ General health } \\
\hline Excellent & 118 & 20.9 & 91.2 & 10.2 & $(89.3,93.0)$ & 92 & 109 & 19.3 & 92.2 & 8.2 & $(90.6,93.8)$ & 92 \\
\hline Very good & 208 & 36.9 & 84.6 & 9.2 & $(83.4,85.9)$ & 92 & 212 & 37.6 & 85.3 & 8.1 & $(84.2,86.4)$ & 85 \\
\hline Good & 161 & 28.5 & 75.8 & 12.3 & $(73.9,77.7)$ & 85 & 169 & 30.0 & 77.2 & 13.7 & $(75.1,79.3)$ & 80 \\
\hline Fair & 62 & 11.0 & 62.4 & 19.8 & $(57.4,67.4)$ & 80 & 61 & 10.8 & 62.8 & 16.7 & $(58.5,67.0)$ & 60 \\
\hline Poor & 15 & 2.7 & 36.9 & 19.7 & $(26.0,47.8)$ & 65 & 13 & 2.3 & 56.5 & 18.6 & $(45.3,67.8)$ & 50 \\
\hline
\end{tabular}

Indonesian EQ-5D-5L norms also plateaued in adjacent, older age groups [17, 18, 47].

The systematically lower mean utility and VAS values noted in online respondents relative to face-to-face respondents were likely affected by a combination of factors. Social desirability bias may have contributed to the observed disparities between samples. A greater proportion of online respondents reported issues across all EQ-5D-5L dimensions compared to face-to-face respondents (Supplementary material Appendices D and E). Further, a smaller portion of online respondents indicated that their general health was excellent compared to the face-to-face respondents $(12.1 \%$ versus 20\%; Table 1). If the populations had similar health, face-to-face respondents may have been unwilling to admit health issues in front of an interviewer due to social desirability bias. Past research also found that self-reported health and norms differed when surveys were self-administered versus interviewer-administered via telephone [48].

However, respondents could also have truly differed between modes of data collection due to the varying selection pressures of recruitment and survey needs for each mode of data collection [49]. US online panels tend to be disproportionately White and unrepresentative of minorities [50, 51]. The company which administered the online surveys used in this study noted comparable patterns of minority under-representation in the online panels they employ, which 


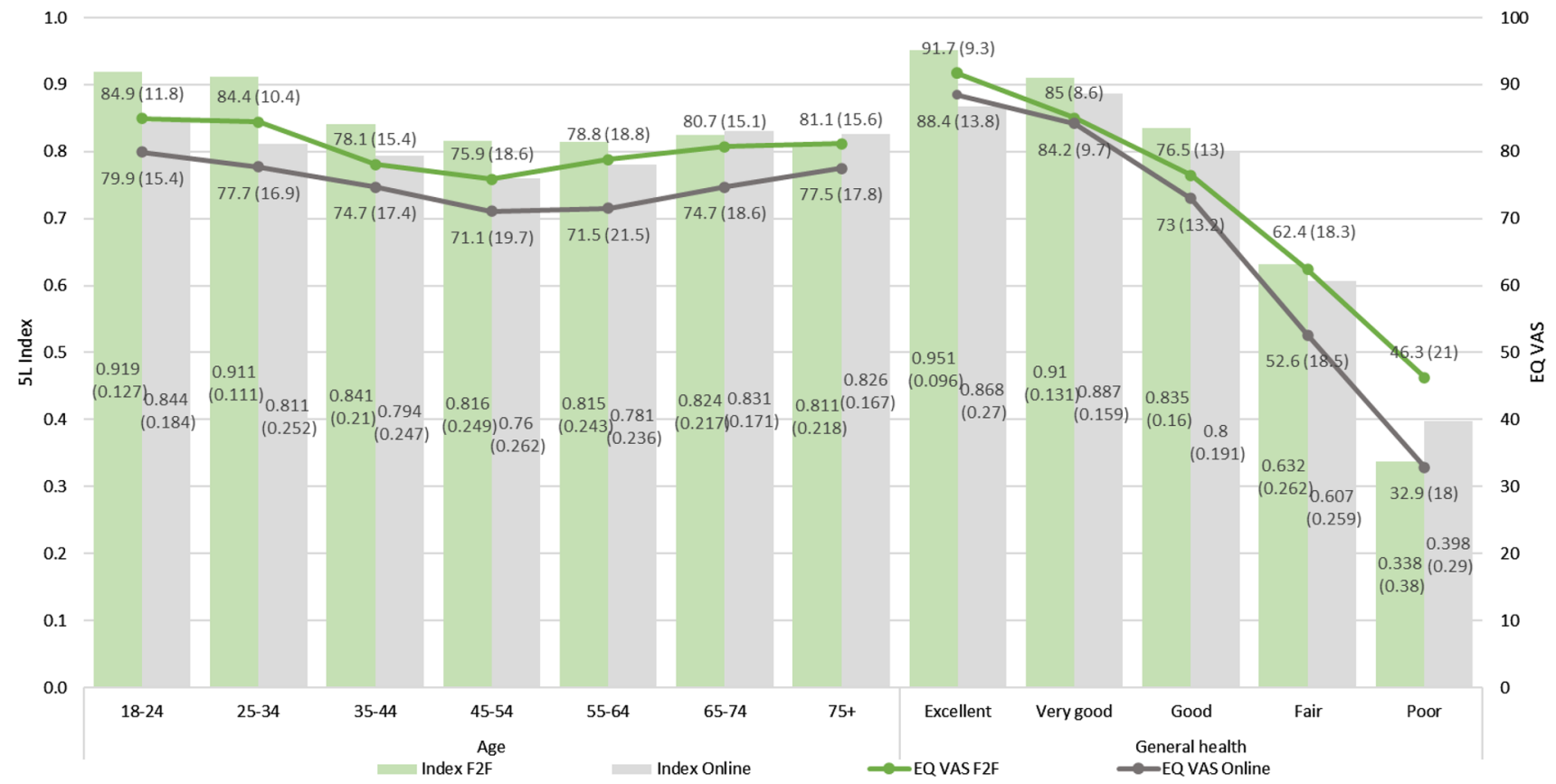

Fig. 1 Face-to-face versus Online mean EQ-5D-5L index and EQ VAS by age band and general health. F2F Face-to-face, VAS visual analogue scale

contributed to the low prevalence of non-Black minorities recruited into the online sample [52]. In addition, online respondents must have reliable access to internet and a computer with which to access it and belong to a survey panel to be selected for the study. Face-to-face respondents needed to participate in the study at centralized locations, potentially requiring transportation to attend interviews outside of their homes. Respondent characteristics which determine agreement to survey participation may additionally differ by mode. Distinctions between samples were most noticeable when respondent characteristics unlikely to be susceptible to social desirability bias were examined. For example, online respondents were more likely to have children under 18 than face-to-face respondents (Table 1). Finally, face-toface respondents aged 65 and older were more comparable to the US general population than these respondents in the online sample, particularly in terms of age and gender distribution. (Supplementary material Appendix G).

As the online population appeared to be less representative of the US general population, the two samples were kept distinct. Based on the aforementioned differences between online and face-to-face respondents as well as the improved representativeness in older respondents, the face-to-face norms should be the primary set of normative values for the US population, particularly for data collected in-person. The online norms may be most useful for comparison of unsupervised data elicited from online panels.

The study had several limitations. Quota-sampling was employed for practical time and cost considerations, and random sampling may have allowed for a more representative sample of the US population, particularly in the faceto-face sample. This shortcoming can be observed in the education attainment and general health of respondents who were at least 65 years old (Supplementary material Appendix G). The sample sizes included in the present study were relatively small in comparison to the US population size, and each face-to-face respondent represented approximately 290,000 US inhabitants. As the EQ-5D-5L is not included in any large-scale US general population surveys, these data were the best available sources to estimate US general population norms. Finally, the mean index and VAS values estimated for each diagnosis in both online and face-to-face samples should only be used as a general guidance for disease burden as these subgroups are likely heterogenous in disease stage, acuity, and patient experience.

With the present study, US EQ-5D-5L utility and EQ VAS norms are now available as general population benchmarks to support health services research across research, clinical, and policy settings for two major methods of data collection. End-users of the EQ-5D-5L may choose the set most appropriate for the application.

Funding Funding was provided by the EuroQol Group. This work was completed while Ruixuan was a graduate student at the University of Illinois at Chicago, during which she was supported by the UIC Dean's Dissertation Fellowship and PhRMA Foundation Pre-Doctoral Health Outcomes Fellowship. 
Data availability Data available upon request were submitted to the corresponding author.

\section{Compliance with ethical standards}

Conflicts of interest All authors are members of the EuroQol group; Ruixuan is currently an employee of Merck Sharp and Dome but was not employed by Merck at the time of study conceptualization, analysis, or manuscript drafting.

Ethics approval Study was conducted on secondary, anonymized data that were previously collected. Ethics approval was not required.

Informed consent Informed consent for study participation was obtained from all individual participants included in the study. Informed consent for publication was obtained from all individual participants included in the study.

Open Access This article is licensed under a Creative Commons Attribution 4.0 International License, which permits use, sharing, adaptation, distribution and reproduction in any medium or format, as long as you give appropriate credit to the original author(s) and the source, provide a link to the Creative Commons licence, and indicate if changes were made. The images or other third party material in this article are included in the article's Creative Commons licence, unless indicated otherwise in a credit line to the material. If material is not included in the article's Creative Commons licence and your intended use is not permitted by statutory regulation or exceeds the permitted use, you will need to obtain permission directly from the copyright holder. To view a copy of this licence, visit http://creativecommons.org/licenses/by/4.0/.

\section{References}

1. Brazier, J., Ratcliffe, J., Salomon, J. A., \& Tsuchiya, A. (2017). Measuring and valuing health benefits for economic evaluation (2nd ed.). Oxford: Oxford University Press.

2. Devlin, N. J., \& Brooks, R. (2017). EQ-5D and the EuroQol group: past, present and future. Applied Health Economics and Health Policy, 15(2), 127-137. https://doi.org/10.1007/s4025 8-017-0310-5.

3. Al-Sayah, F. (2016). Measuring and valuing health: applications of the EQ-5D. https://apersu.ca/wp-content/uploads/2017/07/ Measuring-and-valuing-health-using-the-EQ-5D.pdf. Accessed January 12020.

4. Devlin, N. J. (2016). 5 things you should do with EQ-5D data. https://www.ohe.org/news/5-things-you-should-do-eq-5d-data. Accessed January 12020

5. Huber, M. B., Reitmeir, P., Vogelmann, M., \& Leidl, R. (2016). EQ-5D-5L in the General german population: comparison and evaluation of three yearly cross-section surveys. International Journal of Environmental Research and Public Health, 13(3), 343. https://doi.org/10.3390/ijerph13030343.

6. Yang, F., Devlin, N., \& Luo, N. (2019). Cost-utility analysis using EQ-5D-5L data: does how the utilities are derived matter? Value Health, 22(1), 45-49. https://doi.org/10.1016/j.jval.2018.05.008.

7. Gerlinger, C., Bamber, L., Leverkus, F., Schwenke, C., Haberland, C., Schmidt, G., et al. (2019). Comparing the EQ-5D-5L utility index based on value sets of different countries: impact on the interpretation of clinical study results. BMC Research Notes, 12(1), 18-18. https://doi.org/10.1186/s13104-019-4067-9.
8. Nolan, C. M., Longworth, L., Lord, J., Canavan, J. L., Jones, S. E., Kon, S. S. C., et al. (2016). The EQ-5D-5L health status questionnaire in COPD: validity, responsiveness and minimum important difference. Thorax, 71(6), 493-500. https://doi.org/10.1136/thora xjnl-2015-207782.

9. EQ-5D-5L User Guide-Basic information on how to use the EQ5D-5L instrument (2019). https://euroqol.org/wp-content/uploa ds/2019/09/EQ-5D-5L-English-User-Guide_version-3.0-Sept2019-secured.pdf. Accessed January 12020.

10. Herdman, M., Gudex, C., Lloyd, A., Janssen, M., Kind, P., Parkin, D., et al. (2011). Development and preliminary testing of the new five-level version of EQ-5D (EQ-5D-5L). Quality of Life Research, 20(10), 1727-1736. https://doi.org/10.1007/s1113 6-011-9903-x.

11. Janssen, M. F., Szende, A., Cabases, J., Ramos-Goni, J. M., Vilagut, G., \& Konig, H. H. (2019). Population norms for the EQ-5D-3L: a cross-country analysis of population surveys for 20 countries. The European Journal of Health Economics, 20(2), 205-216. https://doi.org/10.1007/s10198-018-0955-5.

12. Self-Reported Population Health: An International Perspective based on EQ-5D (2014). Dordrecht (NL).

13. Golicki, D., \& Niewada, M. (2017). EQ-5D-5L Polish population norms. Archives of Medical Science, 13(1), 191-200. https://doi. org/10.5114/aoms.2015.52126.

14. Bailey, H., Janssen, M. F., La Foucade, A., \& Kind, P. (2019). EQ-5D-5L population norms and health inequalities for Trinidad and Tobago. PLoS One, 14(4), e0214283. https://doi.org/10.1371/ journal.pone.0214283.

15. Janssen, B., \& Szende, A. (2014). Population norms for the EQ-5D. In A. Szende, B. Janssen, \& J. Cabases (Eds.), Self-reported population health: An international perspective based on (EQ-5D. ed.). Dordrecht: Springer. https://doi. org/10.1007/978-94-007-7596-1_3.

16. McCaffrey, N., Kaambwa, B., Currow, D. C., \& Ratcliffe, J. (2016). Health-related quality of life measured using the EQ5D-5L: South Australian population norms. Health and Quality of Life Outcomes, 14(1), 133. https://doi.org/10.1186/s1295 5-016-0537-0.

17. Purba, F. D., Hunfeld, J. A. M., Iskandarsyah, A., Fitriana, T. S., Sadarjoen, S. S., Passchier, J., et al. (2018). Quality of life of the Indonesian general population: test-retest reliability and population norms of the EQ-5D-5L and WHOQOL-BREF. PLoS One, 13(5), e0197098. https://doi.org/10.1371/journal.pone.0197098.

18. The APERSU Team (2018). Alberta population norms for EQ5D-5L. https://apersu.ca/wp-content/uploads/2018/10/AlbertaNorms-Report_APERSU.pdf. Accessed April 52020.

19. Yang, Z., Busschbach, J., Liu, G., \& Luo, N. (2018). EQ-5D-5L norms for the urban Chinese population in China. Health and Quality of Life Outcomes, 16(1), 210. https://doi.org/10.1186/ s12955-018-1036-2.

20. Burström, K., Johannesson, M., \& Diderichsen, F. (2001). Swedish population health-related quality of life results using the EQ-5D. Quality of Life Research, 10(7), 621-635. https://doi. org/10.1023/A:1013171831202.

21. Hinz, A., Kohlmann, T., Stöbel-Richter, Y., Zenger, M., \& Brähler, E. (2014). The quality of life questionnaire EQ-5D-5L: psychometric properties and normative values for the general German population. Quality of Life Research, 23(2), 443-447. https://doi. org/10.1007/s11136-013-0498-2.

22. Hernandez, G., Garin, O., Pardo, Y., Vilagut, G., Pont, À., Suárez, M., et al. (2018). Validity of the EQ-5D-5L and reference norms for the Spanish population. Quality of Life Research, 27(9), 23372348. https://doi.org/10.1007/s11136-018-1877-5.

23. Gutacker, N., Patton, T., Shah, K., \& Parkin, D. (2020). Using EQ-5D data to measure hospital performance: are general population values distorting patients' choices? Medical Decision 
Making, 40(4), 511-521. https://doi.org/10.1177/0272989X20 927705.

24. Pickard, A. S., Law, E. H., Jiang, R., Pullenayegum, E., Shaw, J. W., Xie, F., et al. (2019). United States valuation of EQ-5D-5L health states using an international protocol. Value in Health. https ://doi.org/10.1016/j.jval.2019.02.009.

25. Oppe, M., Devlin, N. J., van Hout, B., Krabbe, P. F., \& de Charro, F. (2014). A program of methodological research to arrive at the new international EQ-5D-5L valuation protocol. Value Health, 17(4), 445-453. https://doi.org/10.1016/j.jval.2014.04.002.

26. Ramos-Goni, J. M., Oppe, M., Slaap, B., Busschbach, J. J., \& Stolk, E. (2017). Quality control process for EQ-5D-5L valuation studies. Value Health, 20(3), 466-473. https://doi.org/10.1016/j. jval.2016.10.012.

27. Jiang, R., Shaw, J. W., \& Pickard, A. S. (2018). Comaprison of online and face-to-face valuations of the EQ-5D-51 using composite time trade-off and discrete choice tasks. Paper presented at the 40th Annual Meeting of the Society for Medical Decision Making, Montréal, Québec, Canada, October 13-17, 2018

28. Jiang, R., Muehlbacher, A., Shaw, J. W., Lee, T. A., Walton, S., \& Pickard, A. S. (2019). Assessment of the effect of mode of administration on TTO-based preferences. Paper presented at the 10th Meeting "International Academy of Health Preference Research" Basel, Switzerland, 13-14 July, 2019

29. Stolk, E., Ludwig, K., Rand, K., van Hout, B., \& Ramos-Goni, J. M. (2019). Overview, update, and lessons learned from the international EQ-5D-5L valuation work: version 2 of the EQ5D-5L valuation protocol. Value Health, 22(1), 23-30. https:// doi.org/10.1016/j.jval.2018.05.010.

30. Oppe, M., \& van Hout, B. (2017). The "power" of eliciting EQ5D-5L values: the experimental design of the EQ-VT. EuroQol Working Paper Series: EuroQol Research Foundation.

31. Ramos-Goñi, J. M., Oppe, M., Slaap, B., Busschbach, J. J., \& Stolk, E. (2017). Quality control process for EQ-5D-5L valuation studies. Value in Health, 20(3), 466-473.

32. Versteegh, M. M., Attema, A. E., Oppe, M., Devlin, N. J., \& Stolk, E. A. (2013). Time to tweak the TTO: results from a comparison of alternative specifications of the TTO. The European Journal of Health Economics, 14(Suppl 1), S43-51. https://doi.org/10.1007/ s10198-013-0507-y.

33. Zahran, H. S., Kobau, R., Moriarty, D. G., Zack, M. M., Holt, J., \& Donehoo, R. (2005). Health-related quality of life surveillance-United States, 1993-2002. https://www.cdc.gov/mmwr/ preview/mmwrhtml/ss5404a1.htm. Accessed July 202020.

34. WHO GUIDE TO COST-EFFECTIVENESS ANALYSIS (2003). https://www.who.int/choice/publications/p_2003_generalised_ cea.pdf. Accessed July 202020.

35. Major Depression (2019). https://www.nimh.nih.gov/health/stati stics/major-depression.shtml. Accessed April 12020.

36. Any Anxiety Disorder (2017). https://www.nimh.nih.gov/healt h/statistics/any-anxiety-disorder.shtml\#part_155094. Accessed April 12020

37. Scheffler, R., Arnold, D., Qazi, H., Harney, J., Linde, L., Dimick, G., et al. (2018). The anxious generation: the causes and consequences of anxiety disorder among young Americans. https:// gspp.berkeley.edu/assets/uploads/page/Policy_Brief_Final_07161 8.pdf. Accessed April 12020.

38. Greenwood, K., Bapat, V., \& Maughan, M. (2019). Research: people want their employers to talk about mental health. https://hbr. org/2019/10/research-people-want-their-employers-to-talk-about -mental-health. Accessed April 12020.
39. Sampson, C. (2020). Thesis thursday: Hannah Penton. https ://aheblog.com/2020/03/19/thesis-thursday-hannah-penton/. Accessed April 12020.

40. Penton, H. (2019). An investigation into the psychometric performance of existing measures of health, quality of life and wellbeing in older adults. https://etheses.whiterose.ac.uk/24556/. Accessed April 12020.

41. Ratcliffe, J., Lancsar, E., Flint, T., Kaambwa, B., Walker, R., Lewin, G., et al. (2017). Does one size fit all? Assessing the preferences of older and younger people for attributes of quality of life. Quality of Life Research, 26(2), 299-309. https://doi. org/10.1007/s11136-016-1391-6.

42. Segal, D. L., Coolidge, F. L., Mincic, M. S., \& O'Riley, A. (2005). Beliefs about mental illness and willingness to seek help: a crosssectional study. Aging Ment Health, 9(4), 363-367. https://doi. org/10.1080/13607860500131047.

43. Chapter 3: trends in morbidity and risk factors (2018). https:// www.gov.uk/government/publications/health-profile-for-engla nd-2018/chapter-3-trends-in-morbidity-and-risk-factors. Accessed July 202020.

44. Bretschneider, J., Janitza, S., Jacobi, F., Thom, J., Hapke, U., Kurth, T., et al. (2018). Time trends in depression prevalence and health-related correlates: results from population-based surveys in Germany 1997-1999 vs. 2009-2012. BMC Psychiatry, 18(1), 394-394. https://doi.org/10.1186/s12888-018-1973-7.

45. Barclay, R., \& Tate, R. B. (2014). Response shift recalibration and reprioritization in health-related quality of life was identified prospectively in older men with and without stroke. Journal of Clinical Epidemiology, 67(5), 500-507. https://doi.org/10.1016/j. jclinepi.2013.12.003.

46. Sprangers, M. A., \& Schwartz, C. E. (1999). Integrating response shift into health-related quality of life research: a theoretical model. Social Science and Medicine, 48(11), 1507-1515. https ://doi.org/10.1016/s0277-9536(99)00045-3.

47. Poder, T. G., Carrier, N., \& Kouakou, C. R. C. (2020). Quebec health-related quality-of-life population norms using the EQ5D-5L: decomposition by sociodemographic data and health problems. Value Health, 23(2), 251-259. https://doi.org/10.1016/j. jval.2019.08.008.

48. Hanmer, J., Hays, R. D., \& Fryback, D. G. (2007). Mode of administration is important in US national estimates of healthrelated quality of life. Medical Care, 45(12), 1171-1179.

49. Duffy, B., Smith, K., Terhanian, G., \& Bremer, J. (2005). Comparing data from online and face-to-face surveys. International Journal of Market Research, 47(6), 615-639. https://doi. org/10.1177/147078530504700602.

50. Kennedy, C., Mercer, A., Keeter, S., Hatley, N., McGeeney, K., \& Gimenez, A. (2016). Evaluating online nonprobability surveys. https://www.pewresearch.org/methods/2016/05/02/evaluating -online-nonprobability-surveys/. Accessed April 12020.

51. Baker, R. E. G., Blumberg, S. J., Brick, J. M., Couper, M. P., Courtright, M., Dennis, J. M., et al. (2010). AAPOR report on online panels. The Public Opinion Quarterly, 74(4), 711-781.

52. SurveyEngine (2018). Personal communication.

Publisher's Note Springer Nature remains neutral with regard to jurisdictional claims in published maps and institutional affiliations. 